\title{
The parameters of orthotropic sand fabric
}

\author{
LI Xue-feng, SONG fen-fen
}

Solid Mechanics Institute, Ningxia University, Yinchuan 750021, China

KEYWORDS: fabric; orthogonal anisotropy; microstructure; quantitative analysis.

ABSTRACT: The description based on the microscopic theory of sand fabric, the novel amplitude parameters decrypting the spatial arrangement of non-spherical particles is defined, and the verification with the microscopic test for Toyoura sand is conducted. The spatial arrangement of non-spherical particles form the orthotropic of sand which describe with two independent angle of the particles of the long axis direction in three orthogonal directions component, one define three expressions of amplitude parameters on orthotropic plane. This expression is used to describe sand properties of orthotropic, transverse isotropic and isotropic. Experimental results show three amplitude parameters with a clear physical meaning can reflect the variation of orthotropic.

\section{Introduction}

The amplitude parameters research the size of a microscopic fabric orthotropic degree on the surface of metrics. Guo ${ }^{[1]}$, Pradhan ${ }^{[2]}$, etc. a large number of experiments show that sand microscopic anisotropy obviously control the macroscopic strength characteristics; Wan and Guo ${ }^{[3]}$, $\mathrm{Li}$ and Dafalias ${ }^{[4]}$ and a group of scholars all establish the constitutive relationship with fabric tensor; The authors also establish the strength criterion ${ }^{[5]}$, Constitutive model ${ }^{[6]}$, property-dependent plastic potential theory for geomaterials ${ }^{[7]}$, and analyze the mechanics characteristic of the anisotropic sand $^{[8-10]}$. The study of Arthur and Phillips ${ }^{[11]}$, Yamada and Ishihara ${ }^{[12]}$ shown the random arrangement of non-spherical particles and the distribution of pore space own the mathematical laws of probability and statistics, which describes the three causes of sand anisotropy ${ }^{[13,14]}$. As for the quantitative test of amplitude parameters, Yang ${ }^{[15]}$ did the experimental work of quantitative determination fabric for Toyoura sand.

Therefore, in order to determine amplitude parameters, based on the microscopic theory of sand fabric, the novel amplitude parameters descripting the spatial arrangement of non-spherical particles is defined in this paper.

\section{The theory definition of sand fabric tensor}

Non spherical particles random arrangement and its relative void ratio have the fabric correlation properties on the mathematical statistical significance. Oda ${ }^{[13,14]}$ defines the non-spherical particles affect the fabric tensor of the $F_{i j}$ :

$$
F_{i j}=\int_{\Omega} n_{i} n_{j} E(\stackrel{r}{n}) d \Omega(i, j=1,2,3)
$$

Where $E\left({ }^{\prime}\right)$ is the probability density function of the unit vector $n, \quad h$ is the component of non-spherical particles on long axis direction. $E(\stackrel{1}{n})$ can be reformed by use $F_{i j}^{[13,14]}$ :

$$
E(\stackrel{r}{n})=\frac{15}{8 \pi}\left(F_{i j}-\frac{1}{5} \delta_{i j}\right) n_{i} n_{j}
$$

Where $\delta_{i j}$ is Kronecker constant $F_{i j}$ is symmetric. $F_{i j}=F_{j i}$ and $F_{i i} \equiv 1$.

\section{The general expression of fabric tensor}

By the integral relationship with series, integral expression can be equivalent calculation by using the method of superposition of series(1) can be written: 


$$
F_{i j}=\frac{1}{2 N} \sum_{k=1}^{2 N} n_{i}^{(k)} n_{j}^{(k)}(i, j=1,2,3)
$$

Where $N$ is the number of particles in measurement volume; $n_{i}^{(k)}$ and $n_{i}^{(k)}$ represent the component of normal vector of particles long axis on $i$ and $j$ axis respectively. For non-spherical sand particles, the direction of long axis parallel to the grain; For the flat shaped particles, such as clay mineral particles, $\stackrel{n}{n}$ perpendicular to the main plane.Equation (4) as a index describe the anisotropy of non-spherical particle material, it has the correlation characteristics in mathematical laws of statistics. The calculation of the component need to determine the angle between long axis of particle and three axes. $\theta_{1}^{(k)}, \alpha$ is the two angles between the long axis of particle and the coordinate. The direction vector of equation (4) is $\boldsymbol{n}=\left\{\cos \theta_{1}^{(k)}, \sin \theta_{1}^{(k)} \sin \alpha^{(k)}, \sin \theta_{1}^{(k)} \cos \alpha^{(k)}\right\}$.

\section{The definition of orthogonal anisotropic fabric and amplitude parameter}

For orthotropic anisotropic materials, three orthogonal planes should have amplitude parameters in each plane. In the three orthogonal planes $F_{x 1}-F_{x 2}, F_{x 1}-F_{x 3}$ and $F_{x 2}-F_{x 3}$ have an anisotropic amplitude parameter respectively.

According to defined method of amplitude parameters in two-dimensional spatial in this section, we can obtain:

$$
\bar{F}_{1,3}=\frac{1}{2}\left(F_{11}+F_{33}\right) \pm\left[\frac{1}{4}\left(F_{11}-F_{33}\right)^{2}+F_{13}^{2}\right]^{\frac{1}{2}}=\frac{1 \pm a_{1}}{2}
$$

Where

$$
\begin{gathered}
a_{1}=\frac{1}{2 N} \sqrt{\left(\sum_{k=1}^{2 N}\left(\cos ^{2} \theta_{1}^{(k)}-\sin ^{2} \theta_{1}^{(k)} \cos ^{2} \alpha^{(k)}\right)\right)^{2}+\left(\sum_{k=1}^{2 N} \sin 2 \theta_{1}^{(k)} \cos \alpha^{(k)}\right)^{2}} \\
\bar{F}_{1,2}=\frac{1}{2}\left(F_{11}+F_{22}\right) \pm\left[\frac{1}{4}\left(F_{11}-F_{22}\right)^{2}+F_{12}^{2}\right]^{\frac{1}{2}}=\frac{1 \pm a_{2}}{2}
\end{gathered}
$$

Where

$$
\begin{gathered}
a_{2}=\frac{1}{2 N} \sqrt{\left(\sum_{k=1}^{2 N}\left(\cos ^{2} \theta_{1}^{(k)}-\sin ^{2} \theta_{1}^{(k)} \sin ^{2} \alpha^{(k)}\right)\right)^{2}+\left(\sum_{k=1}^{2 N} \sin 2 \theta_{1}^{(k)} \sin \alpha^{(k)}\right)^{2}} \\
\bar{F}_{2,3}=\frac{1}{2}\left(F_{22}+F_{33}\right) \pm\left[\frac{1}{4}\left(F_{22}-F_{33}\right)^{2}+F_{23}^{2}\right]^{\frac{1}{2}}=\frac{1 \pm a_{3}}{2}
\end{gathered}
$$

Where

$$
a_{3}=\frac{1}{2 N} \sqrt{\left(\sum_{k=1}^{2 N}\left(\sin ^{2} \theta_{1}^{(k)} \cos ^{2} 2 \alpha^{(k)}\right)\right)^{2}+\left(\sum_{k=1}^{2 N} \sin ^{2} \theta_{1}^{(k)} \cos 2 \alpha^{(k)}\right)^{2}}
$$

Where $a_{1}, a_{2}$ and $a_{3}$ were the anisotropic amplitude parameters in three orthogonal planes, respectively. Three parameters can be determined by microscopic test. By type (5), (7) type (9), the three parameter value range is 0 to 1 .Three anisotropic amplitude parameters for all non-spherical particles of soil long axis to on the three orthogonal projection of the component, the component with the ideas of the mathematical probability statistics calculation, reflect the irregular shape discrete particle edge direction in the plane of projection value. 


\section{Characteristic analysis of three-dimensional fabric tensor}

\section{The physical significance of amplitude parameters determined by test}

This article selects the Yang ${ }^{[15]}$ microscopic fabric such as quantitative analysis of the results. Test using Toyoura sand, using two sample preparation methods (Macroscopic mechanical properties of two kinds of sample difference is very big, reflects the sample preparation method will produce a lot of anisotropy), solidification sample after processing, slicing, grinding polishing method, by scanning electron microscopy (SEM) for horizontal and vertical direction sample pictures, The picture image analysis, sand microscopic particle analysis results were obtained.

\section{comparative analyses}

Choose two kinds of sample preparation methods, such as Yang ${ }^{[15]}$, the two directions in all four groups microscopic test results, the definition in this paper the anisotropy of amplitude parameter calculation and analysis.

Figure 1 for sanding of amplitude parameter to calculate vertical direction grain of microscopic analysis results. In this paper, particle long axis direction angle and trials righteousness is different. Its relationship to $\theta^{(k)}=\pi / 2-\theta^{(k)}$. Diagram shows $a_{1}, a_{2}, a_{3}$ calculation $\alpha^{(k)}=0$. result, $\alpha^{(k)}=\pi / 2$. $a_{1}$ And $a_{2}$ are the vertical amplitude parameter, calculation results and Yang defined using Oda, such as amplitude formula calculation results trend is the same, different sizes. Figure 1 show that $a_{1}, a_{2}$ and $a_{3}$ vertical, it is indirect test three of this article defined the parameter space of orthogonally. Also parameter calculation of wet strike method in this paper such as amplitude vertical particles microscopic analysis results. $\alpha^{(k)}=\pi / 2$. Calculation results and figure 1 laws, but the overall size is different. The amplitude parameter calculation of sanding and wet strike method vertical grain microscopic analysis results.

Figure1 using observation particle arrangement information, the amplitude of the definition of parameters in this paper computed results; reflect the particles are arranged on the influence of anisotropy amplitude. 




(a)

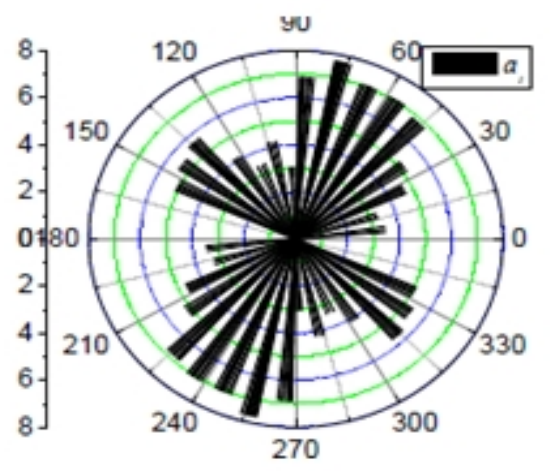

(b)



(c)

Fig. 1 Rose Diagram of Amplitude Parameters. (a) Result of $a_{1}$, (b) Result of $a_{2}$, (b) Result of $a_{3}$

\section{conclusions}

The description based on the theory of non-spherical particle material microscopic fabric, define a new orthotropic and amplitude parameters. Two independent shifty angle can be described on the surface of the particles in the orthogonal expression. Three orthogonal anisotropic amplitude parameters are defined respectively, each of these parameters reflect the degree of anisotropy in the plane of the corresponding. Sand microscopic fabric research provide a more flexible and accurate method.

\section{Acknowledgements}

This work was financially supported by Program of National Natural Science Funds (NSFC through grant No. 5168050) and Ningxia Province Natural Science Funds (No.NX13001). These supports are gratefully acknowledged. 


\section{Reference}

[1] P. J. Guo. "Modified direct shear test for anisotropic strength of sand". Journal of Geotechnical and Geo environmental Engineering, ASCE, 134(9), PP.1311-1318, (2008).

[2] T. B. S.Pradhan, F.Tatsuoka and N.Horii. "Strength and deformation characteristics of sand in torsion simple shear". Soils and Foundations, 28(3), PP.131-148, (1985).

[3] R.G.Wan, P.J.Gua. "A pressure and density dependent dilatancy model for granular materials". Soils and Foundations, 39(6), PP.1-12,_(1999).

[4] X.S.Li, Y.F. Dafalias. "Constitutive modeling of inherently anisotropic sand behavior". Journal of Geotechnical and Geoenvironmental Engineering, ASCE, 128(10), PP.868-880, (2002).

[5] X.F.Li, M.S.Huang and J.G.Qian. "Failure criterion of anisotropic sand with the method of macro-micro incorporation". Chinese Journal of Rock Mechanics and Engineering, 29(9), PP.1885-1892, (2010).

[6] M.S.Huang, X.F.Li and J.G.Qian. "On Strain localization of anisotropic sands". Chinese Journal of Geotechnical Engineering, 34(10), PP.1885-1892, (2012).

[7] X.F.Li, L.Kong and M.S.Huang. "Property-dependent plastic potential theory for geomaterials". Chinese Journal of Geotechnical Engineering, 35(9), PP.1722-1729, (2013).

[8] X.F.Li, M.S.Huang and J.G.Qian. "Analysis of non-coaxial characters of sand forSimple shear test with the method of macro-meson-incorporation". Rock and Soil Mechanics, 34(12), PP. 3417-3424, (2013).

[9] X.F.Li, M.S.Huang and L.Kong. "Failure properties of sand considering rotation of principal stress axis with method of macro-meso incorporation". Rock and Soil Mechanics,34(7), PP.1923-1930, (2013).

[10]X.F.Li, M.S.Huang and J.G.Qian. "Strain localization analysis of Anisotropic Sands based on Non-coaxial Theory”. Engineering mechanics, 31(3), PP.205-211,(2014).

[11]J.R.F. Arthur and A. B.Phillips. "Correspondence: inherent anisotropy in sand". Geotechnique, 22(3), PP. 537-539, (1972).

[12]Y.Yamada and K.Ishihara. "Anisotropic deformation characteristics of sand under three-dimensional stress conditions". Soils andFoundations, 19(2), PP.79-94, (1979).

[13]M.Oda. "Initial fabrics and their relations to mechanical properties of granular materials". Soils and Foundations, 12(1), PP.17-36,(1972).

[14]M.Oda. "Fabric tensor and its geometrical meaning"M.Oda, Iwashitak K, ed. Introduction to mechanics of granular materials, Rotterdam, The Netherlands: A. A. Balkema, PP.27-35(1999).

[15]Z. X.Yang, X. S.Li and J. Yang. "Quantifying and modeling fabric anisotropy of granular soils". Geotechnique, 58(4), PP. 237-248, (2008). 